\title{
Pressure Induced Transitions in Heavy-Fermion Systems ${ }^{\dagger}$
}

\author{
SUn ZHANG ${ }^{a}$ AND Yi-QIAO DONG ${ }^{b}$ \\ ${ }^{a}$ Joint Center for Particle, Nuclear Physics and Cosmology (J-CPNPC), PMO-NJU, Nanjing 210008, China \\ ${ }^{b}$ Key Laboratory of Optical Astronomy, National Astronomical Observatories, Chinese Academy of Sciences, \\ Beijing 100012, China
}

(Received February 13, 2015; in final form July 20, 2015)

\begin{abstract}
In this paper, the electron-type heavy-fermion system and the hole-type heavy-fermion system are classified by means of the cell-volume difference between two typical valence states of $f$ ions. Two kinds of transitions induced by pressure are studied theoretically within a unified picture presented for both two types of heavy-fermion systems. Among these transitions, the incoherence-to-coherence transition in the electron-type heavy-fermion system is confirmed intensively in experiments, while the coherence-to-incoherence transition in the hole-type heavy-fermion system is discussed as a prediction for further observations.
\end{abstract}

DOI: 10.12693 /APhysPolA.128.389

PACS: 75.30.Mb, 75.20.Hr, 71.28. $+\mathrm{d}, 74.62 . \mathrm{Fj}$

\section{Introduction}

Effects of pressure on heavy-fermion (HF) systems have fascinated researchers for a long time not only because of the strong influences of the physical properties under pressure, but also because of the transitions induced by pressure, which reveal some intricate essentials of HF systems [1-14]. HF systems are a class of intermetallic compounds which contain a periodic array of magnetic Kondo ions, involving rare-earth $(4 f)$ or actinide $(5 f)$ elements. The unusual properties of these materials arise mainly from the behavior of their $4 f$ or $5 f$ elements $[15,16]$. It is known that the external pressure is a powerful and clean control parameter for investigating the physics of strongly correlated electron systems [11-14, 17-20].

Moreover, pressure qualitatively acts as a mirror between Ce-based compounds and Yb-based compounds $[17,18,21,22]$. From the ensuing theoretical and experimental works on HF systems, considerable interest has also arisen in the transition induced by pressure: the incoherence-to-coherence transition (ICT) in HF materials, confirmed intensively in $\mathrm{CeCu}_{6}$ [1], $\mathrm{CeInCu}_{2}$ [2-4], and $\mathrm{UBe}_{13}[5,6]$. From the observations, pressure increases the Kondo interaction and the itinerancy of the $f$ electrons, and tends to expand the temperature range for the heavy fermion (or the Fermi liquid) behavior, characterized by $\rho(T)=\rho_{0}+A T^{2}$. The larger quadratic term in temperature accompanies stronger coherence, so pressure establishes coherence in these materials [6] and a transition comes into being [1-6].

One effect of the pressure is to destabilize the larger ion $[5,19-22]$. Then if we investigate the pressuredependent properties on HF systems, the pressure influences from the cell-volume difference between $f$ config-

\footnotetext{
†This work is dedicated to the revered memory of Professor Zheng-Zhong Li
}

urations due to the valence fluctuation of $f$ ions should be taken into account. Pressure favors the $f$ configurations with smaller volume. Based on these, a unified theoretical description is given in this paper for the pressure induced transitions in HF systems. We would like to point out in theory that, besides the transition mentioned above, ICT, there would exist another transition induced by pressure: the coherence-to-incoherence transition (CIT), maybe realized in some Yb-based HF system, such as $\mathrm{YbCu}_{2} \mathrm{Si}_{2}$ or $\mathrm{YbCu}_{4} \mathrm{Ag}$. To the best of our knowledge, such a pressure induced transition has never been discussed before.

\section{Model}

In order to consider the effects of pressure, let us introduce the volume operator. For HF systems, such as $\mathrm{CeAl}_{3}, \mathrm{UBe}_{13}, \mathrm{YbCu}_{2} \mathrm{Si}_{2}$, etc., the most typical valencefluctuation ions $\mathrm{Ce}, \mathrm{U}$ and $\mathrm{Yb}$ can exist in two valence states: one is a singlet, $f^{n}(j=0)$ with zero $j$; the other a magnetic $2 j+1(=N)$-fold-degenerate state, $f^{n+1}(j,+m)$ or $f^{n-1}(j,-m)$ with nonzero $j$. The weak hybridization of conduction electrons with the local $f$ electrons causes the valence to fluctuate by the following changes in the $f$ shell occupation [23-27]:

$f^{n+1}(j,+m) \rightleftharpoons f^{n}(j=0)+e^{-}(j, m)$ for Ce and $\mathrm{U}$,

$f^{n-1}(j,-m) \rightleftharpoons f^{n}(j=0)+h^{+}(j, m)$ for $\mathrm{Yb}$ and Sm. $(2)$ In terms of the Coleman slave-boson (SB) technique [23-25], it is given that

$$
\begin{aligned}
& \left|f^{n} ; j=0\right\rangle_{l} \equiv b_{l}^{\dagger}|0\rangle_{l}, \\
& \left|f^{n \pm 1} ; j, \pm m\right\rangle_{l} \equiv f_{l m}^{\dagger}|0\rangle_{l},
\end{aligned}
$$

where the singlet state of $f$ ions for a site $l$ is represented by an SB field $b_{l}$ and the magnetic multiplet states $f^{n+1}$ and $f^{n-1}$ by a spin- $j$ fermion. The fermion is an electron $\mathrm{e}^{-}$for $\mathrm{Ce}$ and $\mathrm{U}$, while a hole $\mathrm{h}^{+}$for $\mathrm{Yb}$ and $\mathrm{Sm}$, respectively. In this paper, the numbers of channel $N(=2 j+1)=2$ would be taken for simplicity, and two values $( \pm 1 / 2)$ are considered for $m$ here, written as $\sigma$ from now on. Considering the cell-volume difference 
$\Delta \Omega=\Omega_{1}-\Omega_{0}$ between two $f$ configurations, the total volume operator can be written as [28-31]:

$$
\Omega_{t}=\sum_{l} \Omega_{l}=\sum_{l}\left[b_{l}^{\dagger} b_{l} \Omega_{0}+\left(1-b_{l}^{\dagger} b_{l}\right) \Omega_{1}\right],
$$

where $\Omega_{0}$ and $\Omega_{1}$ are the cell volume for the singlet $f^{n}$ $\left(b_{l}^{\dagger} b_{l}=1\right)$ and the multiplet states $f^{n \pm 1}\left(b_{l}^{\dagger} b_{l}=0\right)$, respectively. Evidently, $\Delta \Omega$ is either positive for the cells with the electron-type (e-type) $f$ ions (Ce and $\mathrm{U}$ ) or negative for the cells with the hole-type (h-type) $f$ ions ( $\mathrm{Yb}$ and $\mathrm{Sm})$. Since the more electrons occupy $f$ shells, the larger the ionic radius is.

On the other hand, at low temperature region, the extrinsic effects, including defects and extended impurities, also become dominant and the development of the coherence would be influenced. The impurity effect of $\mathrm{HF}$ systems discussed in this paper can be looked as disorder doping on the $f$-electron band [32-38], and the nonmagnetic La-like atoms without $f$ electrons (A atoms) substitute the Ce-like or Yb-like atoms with $f$ electrons (B atoms). Such compositional disorder HF systems include $\mathrm{Ce}_{1-x} \mathrm{La}_{x} \mathrm{Cu}_{6}[32,33], \mathrm{Ce}_{1-x} \mathrm{La}_{x} \mathrm{Cu}_{2} \mathrm{Si}_{2}$ [34, 35], and $\mathrm{Ce}_{1-x} \mathrm{La}_{x} \mathrm{Al}_{3}$ [36].

Using the random variable

$$
\xi_{l}= \begin{cases}1 & \text { for } l \in A, \\ 0 & \text { for } l \in B,\end{cases}
$$

the disorder Hamiltonian of the system in the SB formalism reads

$$
\begin{aligned}
H & =\sum_{\boldsymbol{k} \sigma}\left[\varepsilon_{\boldsymbol{k}} c_{\boldsymbol{k} \sigma}^{\dagger} c_{\boldsymbol{k} \sigma}+\left(\alpha \varepsilon_{\boldsymbol{k}}-E_{0}\right) f_{\boldsymbol{k} \sigma}^{\dagger} f_{\boldsymbol{k} \sigma}\right] \\
& +\sum_{l \sigma} \xi_{l}\left(E_{L}+E_{0}\right) f_{l \sigma}^{\dagger} f_{l \sigma} \\
& +V \sum_{l \sigma}\left(1-\xi_{l}\right)\left(c_{l \sigma}^{\dagger} f_{l \sigma} b_{l}^{\dagger}+f_{l \sigma}^{\dagger} c_{l \sigma} b_{l}\right),
\end{aligned}
$$

with a constraint

$$
\sum_{\sigma} f_{l \sigma}^{\dagger} f_{l \sigma}+b_{l}^{\dagger} b_{l}=1 \quad \text { for } l \in B,
$$

where $\left(-E_{0}\right)$ and $E_{L}$ are the energies for $f$ electrons on magnetic (B) and nonmagnetic (A) atoms, respectively. Other notations are standard. Then it describes the Yoshimori-Kasai model (YKM) [37, 38] for HF systems with $\alpha \ll 1(\neq 0)$, and the partition function of the system with the constraint condition (8) can be written as

$$
\begin{aligned}
Z & =\int_{-\pi / \beta}^{\pi / \beta}\left[\prod_{l} \frac{\beta \mathrm{d} \lambda_{l}}{2 \pi}\right] \int D\left[b, b^{\dagger}\right] \int D\left[f, f^{\dagger} ; c, c^{\dagger}\right] \\
& \times \exp \left(-\int_{0}^{\beta} \mathrm{d} \tau L(\tau)\right),
\end{aligned}
$$

where

$$
\begin{gathered}
L(\tau)=\sum_{l} b_{l}^{\dagger} \frac{\mathrm{d}}{\mathrm{d} \tau} b_{l}+\sum_{\boldsymbol{k} \sigma} c_{\boldsymbol{k} \sigma}^{\dagger} \frac{d}{d \tau} c_{\boldsymbol{k} \sigma}+\sum_{l \sigma} f_{l \sigma}^{\dagger} \frac{d}{d \tau} f_{l \sigma} \\
+H+p \Omega_{t}+i \sum_{l} \lambda_{l}\left(1-\xi_{l}\right)\left(\sum_{\sigma} f_{l \sigma}^{\dagger} f_{l \sigma}+b_{l}^{\dagger} b_{l}-1\right)
\end{gathered}
$$

and $\Omega_{t}$ is the total volume operator in the impurity case $[30,31]$ :

$$
\Omega_{t}=\sum_{l}\left\{\xi_{l} \Omega_{L}+\left(1-\xi_{l}\right)\left[b_{l}^{\dagger} b_{l} \Omega_{0}+\left(1-b_{l}^{\dagger} b_{l}\right) \Omega_{1}\right]\right\},
$$

with $\Omega_{L}$ the cell volume of an impurity site (A site), $\Omega_{0}$ and $\Omega_{1}$ for a $\mathrm{B}$ site. Following Read and Newns [26, 27], we perform the gauge transformations

$$
\begin{aligned}
& b_{l}(\tau)=r_{l}(\tau) \exp \left(\mathrm{i}\left(1-\xi_{l}\right) \theta(\tau)\right), \\
& f_{l \sigma}(\tau)=f_{l \sigma}^{\prime}(\tau) \exp \left(\mathrm{i}\left(1-\xi_{l}\right) \theta(\tau)\right), \\
& c_{\boldsymbol{k} \sigma}(\tau)=c_{\boldsymbol{k} \sigma}^{\prime}(\tau), \\
& \lambda_{l}^{\prime}(\tau)=\lambda_{l}+\dot{\theta}(\tau),
\end{aligned}
$$

to obtain a new functional integral (dropping primes)

$$
\begin{aligned}
Z & =\int_{-\pi / \beta}^{\pi / \beta}\left[\prod_{l} \frac{\beta \mathrm{d} \lambda_{l}}{2 \pi}\right] \int\left[\prod_{\tau} r(\tau) \mathrm{D} r\right] \int D\left[b, b^{\dagger}\right] \\
& \times \int D\left[f, f^{\dagger} ; c, c^{\dagger}\right] \exp \left(-\int_{0}^{\beta} d \tau L^{\prime}(\tau)\right),
\end{aligned}
$$

where

$$
\begin{aligned}
& L^{\prime}(\tau)=\sum_{\boldsymbol{k} \sigma} c_{\boldsymbol{k} \sigma}^{\dagger}\left(\frac{\mathrm{d}}{\mathrm{d} \tau}+\varepsilon_{\boldsymbol{k}}\right) c_{\boldsymbol{k} \sigma} \\
& \quad+\sum_{l \sigma} f_{l \sigma}^{\dagger}\left[\frac{\mathrm{d}}{\mathrm{d} \tau}+\xi_{l}\left(E_{L}+E_{0}\right)+\mathrm{i} \lambda_{l}\left(1-\xi_{l}\right)\right] f_{l \sigma} \\
& \quad+\sum_{\boldsymbol{k} \sigma} f_{\boldsymbol{k} \sigma}^{\dagger}\left(\alpha \varepsilon_{\boldsymbol{k}}-E_{0}\right) f_{\boldsymbol{k} \sigma} \\
& \quad+\sum_{l \sigma} r_{l} V\left(1-\xi_{l}\right)\left(c_{l \sigma}^{\dagger} f_{l \sigma}+f_{l \sigma}^{\dagger} c_{l \sigma}\right) \\
& \quad+\sum_{l}\left[\left(\mathrm{i} \lambda_{l}-p \Delta \Omega\right)\left(1-\xi_{l}\right)\left(r_{l}^{2}-1\right)\right. \\
& \left.\quad+p\left(1-\xi_{l}\right) \Omega_{0}+p \xi_{l} \Omega_{L}\right] .
\end{aligned}
$$

Retaining the lattice periodicity, we replace $\mathrm{i} \lambda_{l}(\tau)$ by $E_{f}+E_{0} \equiv \lambda$ and $r(\tau)$ by $r$. Then the effective Hamiltonian in the SB mean-field (MF) approximation is obtained

$$
\begin{aligned}
& H_{\mathrm{eff}}=\sum_{\boldsymbol{k} \sigma}\left[\varepsilon_{\boldsymbol{k}} c_{\boldsymbol{k} \sigma}^{\dagger} c_{\boldsymbol{k} \sigma}+\left(\alpha \varepsilon_{\boldsymbol{k}}+E_{f}\right) f_{\boldsymbol{k} \sigma}^{\dagger} f_{\boldsymbol{k} \sigma}\right] \\
& +r V \sum_{l \sigma}\left(c_{l \sigma}^{\dagger} f_{l \sigma}+f_{l \sigma}^{\dagger} c_{l \sigma}\right)+\sum_{l \sigma} \xi_{l}\left[\varepsilon_{L} f_{l \sigma}^{\dagger} f_{l \sigma}\right. \\
& \left.-r V\left(c_{l \sigma}^{\dagger} f_{l \sigma}+f_{l \sigma}^{\dagger} c_{l \sigma}\right)\right]+(1-x) N_{s} \lambda\left(r^{2}-1\right) \\
& +x N_{s} p \Omega_{L}+(1-x) N_{s} p\left[\Omega_{0}+\left(1-r^{2}\right) \Delta \Omega\right],
\end{aligned}
$$

where $\varepsilon_{L}=E_{L}-E_{f}$ and $x=\left(1 / N_{s}\right) \sum_{l} \xi_{l}, N_{s}$ is the total number of sites in the system. Then the volume operator (11) can be written as

$$
\begin{gathered}
\Omega_{t}=x \Omega_{L}+(1-x)\left[r^{2} \Omega_{0}+\left(1-r^{2}\right) \Omega_{1}\right]= \\
x \Omega_{L}+(1-x)\left[\Omega_{0}+\left(1-r^{2}\right) \Delta \Omega\right] .
\end{gathered}
$$

To apply the coherent potential approximation (CPA) [39, 40], the coherent potential can be assumed as a $2 \times 2$ matrix [41-44]: 


$$
S(\omega, x)=\left(\begin{array}{ll}
0 & r V \\
r V & S_{f f}
\end{array}\right),
$$

and the self-consistent condition in single-site CPA is [45]:

$$
x t_{\mathrm{A}}+(1-x) t_{\mathrm{B}}=0,
$$

where

$$
\begin{aligned}
& t_{\mathrm{A}}=\frac{1}{F_{f f}}\left(\begin{array}{ll}
0 & 0 \\
0 & -1
\end{array}\right), \\
& t_{\mathrm{B}}=\frac{1}{1+S_{f f} F_{f f}}\left(\begin{array}{ll}
0 & 0 \\
0 & -S_{f f}
\end{array}\right) .
\end{aligned}
$$

Then

$$
S_{f f} F_{f f}=-x,
$$

and Hamiltonian in the CPA formalism is

$$
\begin{aligned}
H & =\sum_{\boldsymbol{k} \sigma}\left[\varepsilon_{\boldsymbol{k}} c_{\boldsymbol{k} \sigma}^{\dagger} c_{\boldsymbol{k} \sigma}+\left(\alpha \varepsilon_{\boldsymbol{k}}+E_{f}+S_{f f}\right) f_{\boldsymbol{k} \sigma}^{\dagger} f_{\boldsymbol{k} \sigma}\right. \\
& \left.+r V\left(c_{\boldsymbol{k} \sigma}^{\dagger} f_{\boldsymbol{k} \sigma}+f_{\boldsymbol{k} \sigma}^{\dagger} c_{\boldsymbol{k} \sigma}\right)\right]+x N_{s} p \Omega_{L} \\
& +(1-x) N_{s} \lambda\left(r^{2}-1\right)+(1-x) N_{s} p \\
& \times\left[\Omega_{0}+\left(1-r^{2}\right) \Delta \Omega\right] .
\end{aligned}
$$

Applying the Hellmann-Feynman theorem as in Refs. [26, 27], we obtain two key relations

$$
\begin{aligned}
& (1-x)\left(1-r^{2}\right)=-\frac{2}{\pi} \int_{-\infty}^{\infty} \mathrm{d} \omega f(\omega) \operatorname{Im} F_{f f}\left(\omega+\mathrm{i} 0^{+}\right), \\
& (1-x) r(\lambda-p \Delta \Omega)=\frac{2 V}{\pi} \int_{-\infty}^{\infty} \mathrm{d} \omega f(\omega) \operatorname{Im} F_{f c}\left(\omega+\mathrm{i} 0^{+}\right),
\end{aligned}
$$
together with the average site Green functions (GF):

$$
\begin{aligned}
& F_{c c}(\omega)= \\
& \quad \frac{1}{N_{s}} \sum_{\boldsymbol{k}} \frac{\omega-\alpha \varepsilon_{\boldsymbol{k}}-E_{f}-S_{f f}}{\left(\omega-\varepsilon_{\boldsymbol{k}}\right)\left(\omega-\alpha \varepsilon_{\boldsymbol{k}}-E_{f}-S_{f f}\right)-(r V)^{2}}, \\
& F_{c f}(\omega)=F_{f c}(\omega)= \\
& \quad \frac{1}{N_{s}} \sum_{\boldsymbol{k}} \frac{r V}{\left(\omega-\varepsilon_{\boldsymbol{k}}\right)\left(\omega-\alpha \varepsilon_{\boldsymbol{k}}-E_{f}-S_{f f}\right)-(r V)^{2}}, \\
& F_{f f}(\omega)= \\
& \quad \frac{1}{N_{s}} \sum_{\boldsymbol{k}} \frac{\omega-\varepsilon_{\boldsymbol{k}}}{\left(\omega-\varepsilon_{\boldsymbol{k}}\right)\left(\omega-\alpha \varepsilon_{\boldsymbol{k}}-E_{f}-S_{f f}\right)-(r V)^{2}},
\end{aligned}
$$

to constitute a set of self-consistent equations based on CPA.

The $f$-electron density of states ( $f$-DOS) per magnetic (B) site for each spin is calculated from

$$
N_{f}(\omega, p \Delta \Omega, x)=-\frac{1}{\pi(1-x)} \operatorname{Im} F_{f f}\left(\omega+\mathrm{i} 0^{+}\right),
$$

based on the assumption of the unperturbed DOS of conduction band

$$
N_{0}(\omega)=\frac{2}{\pi D} \sqrt{1-\left(\frac{\omega}{D}\right)^{2}} \Theta(D-|\omega|),
$$

where $\Theta(x)$ is the step function and $D$ - the half-width of the unperturbed conduction band.
For a doped HF system, when the concentration of $f$ ions $(1-x)$ increases, the itinerancy of the $f$ electrons enhances due to the growing coherent scattering. The system would form a coherent lattice state of a global Kondo singlet in the high concentration region $[(1-x) \rightarrow$ 1] replacing the collection of independent Kondo impurity singlets in the dilute limit $[(1-x) \rightarrow 0]$ [41-44]. After the concentration reaches a critical value $(1-x)_{c}=0.7$ for our choice of the parameter values here, the curve of $f$-DOS changes into the concave shape from a convex one, showing the ICT induced by doping from a Kondo impurity resonant state with single-peak structure into a Kondo coherent state with two-peak pseudogap structure. It is known that such an alloying effect induced by doping is in agreement with experiments qualitatively [32, 33].

Now let us discuss the effects of pressure.

I) ICT in the e-type HF systems induced by pressure. From Fig. 1a and b, for the e-type HF systems $(\Delta \Omega>0)$, such as $\mathrm{CeCu}_{2} \mathrm{Si}_{2}[17,18], \mathrm{CeCu}_{6}$ [1], and $\mathrm{UBe}_{13}[5,6]$, pressure increases the correlation between $f$ ions and as well the hybridization between $f$ and conduction bands, and tends to enhance the itinerancy of $f$ electrons and the coherence of the system $[1-6,21,22]$. At $1-x=0.65$ (the solid lines in Fig. $1 \mathrm{a}$ and $\mathrm{b}$ ), the $f$-DOS still displays with a single-peak structure $(p=0)$, showing the character of the Kondo impurity resonant state. When pressure is applied $(p \Delta \Omega=0.25 D)$, the two-peak pseudogap appears, corresponding to the coherent state formation, and ICT takes place. Such a transition is confirmed by the results of the electrical resistivity in Fig. $2 \mathrm{a}-\mathrm{c}$, which is calculated based on the Kubo formula in CPA formalism [40]:

$$
\begin{aligned}
& \sigma(T, p, x)=\frac{2 e^{2} v_{\mathrm{F}}^{2}}{3 \pi \hbar^{2} \Omega} \int_{-\infty}^{\infty} \mathrm{d} \omega\left(-\frac{\partial f}{\partial \omega}\right) \\
& \times \sum_{\boldsymbol{k}}\left[\operatorname{Im} \bar{G}_{c c}\left(\boldsymbol{k}, p, \omega+\mathrm{i} 0^{+}\right)\right]^{2},
\end{aligned}
$$

where $v_{\mathrm{F}}$ is the Fermi velocity, $\Omega$ the volume of the system and

$$
\begin{aligned}
& \bar{G}_{c c}(\boldsymbol{k}, p, \omega)= \\
& \quad \frac{\omega-\alpha \varepsilon_{\boldsymbol{k}}-E_{f}-S_{f f}}{\left(\omega-\varepsilon_{\boldsymbol{k}}\right)\left(\omega-\alpha \varepsilon_{\boldsymbol{k}}-E_{f}-S_{f f}\right)-(r V)^{2}}
\end{aligned}
$$

is the matrix element of the effective medium GF for conduction electrons. In order to extract the information of HF (or the Fermi liquid) behavior, the low-temperature region of the result in Fig. $2 \mathrm{a}$ is used to fit the formula $\rho-\rho_{0}=A T^{2}$. The coefficient (or the slope of the line) $A$ shown in Fig. 2c changes its sign from negative to positive by the application of pressure, indicating the transition from the incoherent state to a coherent state of the system $[32,33]$. From experiments, pressure tends to establish the coherence in the e-type $\mathrm{HF}$ materials, such as $\mathrm{CeCu}_{6}$ [1], $\mathrm{CeInCu}_{2}$ [2-4], $\mathrm{UBe}_{13}[5,6]$, etc. Under high pressure, these systems have been investigated in detail, and the crossover between incoherent and coherent scattering is studied in a controlled way by variation of pressure. 


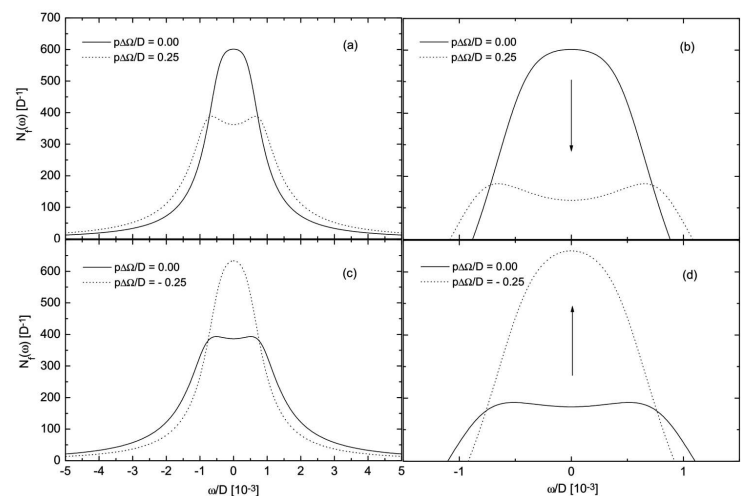

Fig. 1. $f$-DOS of the $e$-type HF system [(a) and (b)] at $1-x=0.65$ and of the $h$-type HF system [(c) and (d)] at $1-x=0.75$ under applied pressure. The parameters for the numerical calculation are chosen as $V^{2}=0.3 D^{2}$, $E_{0}=1.12 D$, and $\eta=1.03$. For clearness, (b) and (d) are the enlarged ones of the significant part of (a) and (c) respectively, and the arrow shows the direction of the transition induced by pressure.

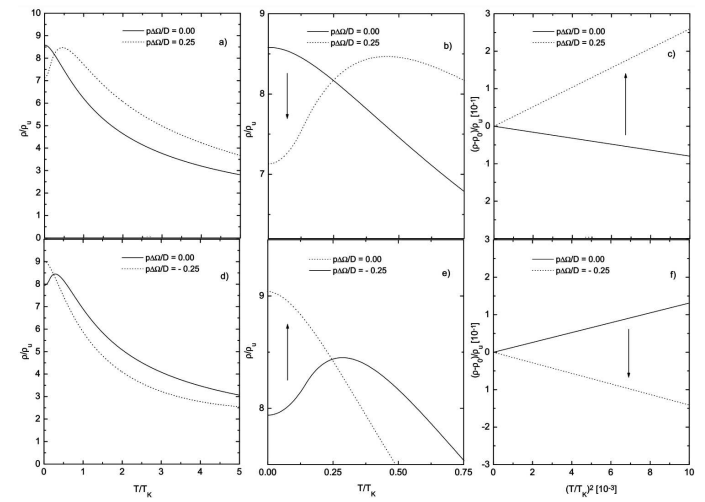

Fig. 2. The electrical resistivity $\rho$ of the e-type HF system [(a), (b), and (c)] and of the $h$-type HF system $[(\mathrm{d}),(\mathrm{e})$, and $(\mathrm{f})]$ under applied pressure. The parameters for the numerical calculation are the same as those in Fig. 1 and $\rho_{u}=3 \pi \hbar^{2} D^{2} \Omega / 2 e^{2} v_{\mathrm{F}}^{2}$. The pressure effect on the Kondo temperature has been taken into account. For clearness, (b) and (e) are the enlarged ones of the significant part of (a) and (d) respectively, and the arrow shows the direction of the transition induced by pressure. While (c) and (f) give the change of the coefficient $\mathrm{A}$ for (a) and (d) respectively.

II) CIT in the h-type HF systems induced by pressure. From Fig. $1 c$ and d, for the $h$-type HF systems $(\Delta \Omega<0)$, such as $\mathrm{YbCu}_{2} \mathrm{Si}_{2}$ [17-20], pressure decreases the correlation between $f$ ions and the hybridization between $f$ and conduction bands [21, 22], and tends to suppress the coherence of the system [17-20]. At $1-x=0.75$ (the solid lines in Fig. $1 \mathrm{c}$ and $\mathrm{d}$ ), the $f$-DOS is already the two-peak pseudogap formation $(p=0)$, showing the coherent character of the system. When pressure is applied $(p \Delta \Omega=-0.25 D)$, the pseudogap disappears, replaced by a single-peak resonant structure, indicating the coherence-to-incoherence transition of the system. Such a CIT is confirmed from the results of resistivity in Fig. 2d-f. The coefficient $A$ changes its sign from positive to negative under pressure (Fig. 2f), showing the transition from the coherent state to an incoherent state of the system. Although such a CIT has not been mentioned before, we hope that it might be observed in some $h$-type HF materials, e.g., $\mathrm{YbCu}_{2} \mathrm{Si}_{2}, \mathrm{YbCuAl}$, or $\mathrm{YbAgCu}_{4}$.

\section{Summary}

In summary, two kinds of pressure induced transitions in $\mathrm{HF}$ systems are studied in the vicinity of the critical point. One of them, ICT in the e-type HF systems is confirmed in experiments, while the other, CIT in the h-type HF systems might be observed in further measurements. It is also found that pressure acts qualitatively as a mirror between the e-type HF systems and the h-type HF systems $[17,18]$. A transition induced by pressure in the e-type HF systems could be looked as a mirror image of the transition in their h-type counterparts, and vice versa.

\section{References}

[1] J.D. Thompson, Z. Fisk, Phys. Rev. B 31, 389 (1985).

[2] T. Kagayama, G. Oomi, H. Takahashi, N. Mōri, Y. Onuki, T. Komatsubara, Phys. Rev. B 44, 7690 (1991).

[3] T. Kagayama, G. Oomi, H. Takahashi, N. Mōri, Y. Ōnuki, T. Komatsubara, J. Alloys Comp. 181, 185 (1992).

[4] T. Kagayama, G. Oomi, R. Yagi, Y. Iye, Y. Ōnuki, T. Komatsubara, J. Alloys Comp. 207-208, 271 (1994).

[5] M.W. McElfresh, M.B. Maple, J.O. Willis, Z. Fisk, J.L. Smith, J.D. Thompson, Phys. Rev. B 42, 6062 (1990).

[6] M.C. Aronson, J.D. Thompson, J.L. Smith, Z. Fisk, M.W. McElfresh, Phys. Rev. Lett. 63, 2311 (1989).

[7] J.C. Cooley, M.C. Aronson, Z. Fisk, P.C. Canfield, Phys. Rev. Lett. 74, 1629 (1995).

[8] J.C. Cooley, M.C. Aronson, Z. Fisk, P.C. Canfield, Physica B 199-200, 486 (1994).

[9] A.V. Goltsev, M.M. Abd-Elmeguid, J. Phys. Condens. Matter 17, S813 (2005).

[10] T. Watanuki, D. Kawana, A. Machida, A.P. Tsai, J. Phys. Soc. Jpn. 80, SA087 (2011).

[11] S. Seo, V.A. Sidorov, H. Lee, D. Jang, Z. Fisk, J.D. Thompson, T. Park, Phys. Rev. B 85, 205145 (2012).

[12] S. Ohara, T. Yamashita, T. Shiraishi, K. Matsubayashi, Y. Uwatoko, J. Phys. Conf. Ser. 400, 042048 (2012).

[13] P. Logg, Z. Feng, T. Ebihara, Y. Zou, S. Friedemann, P. Alireza, S. Goh, F. Malte Grosche, Phys. Status Solidi B 250, 515 (2013). 
[14] N. Kawamura, R. Sasaki, K. Matsubayashi, N. Ishimatsu, M. Mizumaki, Y. Uwatoko, S. Ohara, S. Watanabe, J. Phys. Conf. Ser. 568, 042015 (2014).

[15] M. Dzero, V. Galitski, J. Exp. Theor. Phys. 117, 499 (2013).

[16] S. Burdin, C. Lacroix, Phys. Rev. Lett. 110, 226403 (2013).

[17] D. Jaccard, H. Wilhelm, K. Alami-Yadri, E. Vargoz, Physica B 259-261, 1 (1999).

[18] D. Jaccard, E. Vargoz, K. Alami-Yadri, H. Wilhelm, Rev. High Pressure Sci. Technol. 7, 412 (1998).

[19] K. Alami-Yadri, H. Wilhelm, D. Jaccard, Solid State Commun. 108, 279 (1998).

[20] K. Alami-Yadri, H. Wilhelm, D. Jaccard, Physica B 259-261, 157 (1999).

[21] J.D. Thompson, H.A. Borges, Z. Fisk, S. Horn, R.D. Parks, G.L. Wells, in: Theoretical and Experimental Aspects of Valence Fluctuations and Heavy Fermions, Eds. L.C. Gupta, S.K. Malik, Plenum, New York 1987, p. 151.

[22] J.D. Thompson, in: Selected Topics in Magnetism, Eds. L.C. Gupta, M.S. Multani, World Sci., Singapore 1993, p. 107.

[23] P. Coleman, Phys. Rev. B 29, 3035 (1984).

[24] P. Coleman, J. Magn. Magn. Mater. 47-48, 323 (1985).

[25] P. Coleman, Phys. Rev. B 35, 5072 (1987).

[26] D.M. Newns, N. Read, Adv. Phys. 36, 799 (1987).

[27] N. Read, D.M. Newns, Solid State Commun. 52, 993 (1984).

[28] X.H. Xu, Z.Z. Li, M.W. Xiao, Phys. Rev. B 54, 12993 (1996).
[29] H. Lin, Z.Z. Li, M.W. Xiao, X.H. Xu, Commun. Theor. Phys. 31, 49 (1999).

[30] S. Zhang, Z.Z. Li, W. Xu, X.H. Xu, Commun. Theor. Phys. 34, 11 (2000).

[31] S. Zhang, Phys. Rev. B 63, 224402 (2001).

[32] A. Sumiyama, Y. Oda, H. Nagano, Y. Ōnuki, K. Shibutani, T. Komatsubara, J. Phys. Soc. Jpn. 55, 1294 (1986).

[33] Y. Ōnuki, T. Komatsubara, J. Magn. Magn. Mater. 63-64, 281 (1987).

[34] Y. Ōnuki, T. Hirai, T. Kumazawa, T. Komatsubara, Y. Oda, J. Phys. Soc. Jpn. 56, 1454 (1987).

[35] F.G. Aliev, N.B. Brandt, V.V. Moshchalkov, S.M. Chudinov, J. Low. Temp. Phys. 37, 61 (1984).

[36] R. Pietri, K. Ingersent, B. Andraka, Phys. Rev. Lett. 86, 1090 (2001).

[37] A. Yoshimori, H. Kasai, J. Magn. Magn. Mater. 31-34, 475 (1983).

[38] A. Yoshimori, H. Kasai, Solid State Commun. 58, 259 (1986).

[39] B. Velick, S. Kirkpatrick, H. Ehrenreich, Phys. Rev. 175, 747 (1968).

[40] F. Brouers, A.V. Vedyayev, Phys. Rev. B 5, 348 (1972).

[41] Z.Z. Li, Y. Qiu, Phys. Rev. B 43, 12906 (1991).

[42] Z.Z. Li, W. Xu, C. Chen, M.W. Xiao, Phys. Rev. B 50, 11332 (1994).

[43] S. Zhang, Phys. Rev. B 65, 064407 (2002).

[44] S. Zhang, Phys. Lett. A 284, 49 (2001).

[45] F. Yonezawa, in: The Structure and Properties of Matter, Ed. T. Matsubara, Springer, Berlin 1982, Ch. 11. 